\title{
Cavity Enhanced Absorption Spectroscopy Sensor
}

\author{
M. Nowakowski, J. Wojtas, Z. Bielecki* and J. MikołajczyK \\ Institute of Optoelectronics at Military University of Technology, S. Kaliskiego 2, 00-908 Warszawa, Poland \\ The paper presents opportunities of cavity enhanced absorption spectroscopy technique application in \\ nitrogen oxides $\left(\mathrm{NO}_{x}\right)$ detection. In this method the concentration of an absorbing gas is determined by measure \\ of decay time of the light pulse trapped in an optical cavity. The measurements are not sensitive to fluctuation \\ of both laser power and photodetector sensitivity. The cavity enhanced absorption spectroscopy technique is a \\ modification of cavity ring down spectroscopy technique where the off-axis adjustment of the resonator is used. \\ $\mathrm{NO}_{x}$ detection is carried out in the visible and infrared range. The signal is registered with a developed low noise \\ photoreceiver. Features of the presented sensor show that it is possible to build a portable trace gases sensor. Its \\ sensitivity could be comparable with chemical detectors. Such a system has several advantages: relatively low \\ price, small size and weight, and detection possibility of other gases.
}

PACS numbers: 07.50.Qx, 42.79.Sz, 43.60.-c, 07.07.Df

\section{Introduction}

Direct absorption spectroscopy is a simple, noninvasive, in situ technique for obtaining information about different species. From an absorption spectrum, quantitative absolute concentrations can be extracted. However, direct absorption spectroscopy suffers from a low sensitivity that limits its extension into several research fields. This low sensitivity results from the fact that a small light attenuation has been measured. Several experimental setups can improve the sensitivity, increasing the absorption path length, and combining direct absorption spectroscopy with a modulation technique. In 1988 $\mathrm{O}^{\prime}$ Keefe and Deacon developed a pulsed absorption cavity ring down spectroscopy (CRDS) technique. In the last decade there were many various experimental schemes of CRDS spectroscopy [1].

Cavity ring down spectroscopy has become a widely used technique in the optical absorption analysis of atoms, molecules, and optical components. The technique allows the determination of total optical losses within a closed cavity comprised of two or more mirrors. CRDS is a technique capable of making quantitative diagnostic of trace species in a gaseous environment.

\section{Basic analysis of the CRDS method}

The principle of CRDS method is based upon a measurement of the decay time of radiation trapped in optical resonator with a high quality factor. Figure 1 shows scheme of CRDS setup.

A laser pulse of about $50 \mathrm{~ns}$ duration is coupled into cavity, consisting of two high-reflective mirrors

\footnotetext{
* corresponding author; e-mail: zbielecki@wat.edu.pl
}

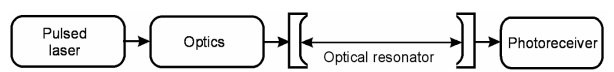

Fig. 1. Scheme representation of the CRDS method.

$(R>99.995 \%)$. At each mirror a small part $(1-R)$ of the circulating radiation will be coupled out, while the remaining part is reflected back in the cavity (Fig. 2).

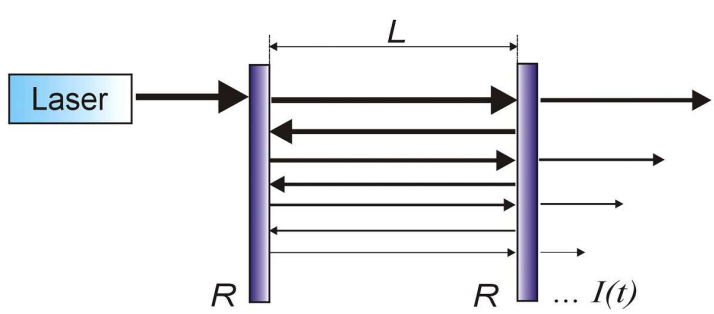

Fig. 2. Idea of CRDS operation.

A small fraction of this radiation transmits through each mirror with each pass. This radiation is detected at the output mirror with a photomultiplier and next it is monitored on a digital oscilloscope. If an absorbing species is placed in the cavity the decay time will be shortened at that wavelength where absorption occurs. The decay time is indirectly proportional to the absorbing coefficient of the species. The decay time of radiation is measured once when the cavity is empty (Fig. 3, dashed line B) and next when the cavity is filled with the absorber (Fig. 3, line A).

If there are additional losses inside the cavity due the presence of absorbing and light scattering species, the light intensity inside the cavity will still decay exponentially provided the absorption follows Beer law 


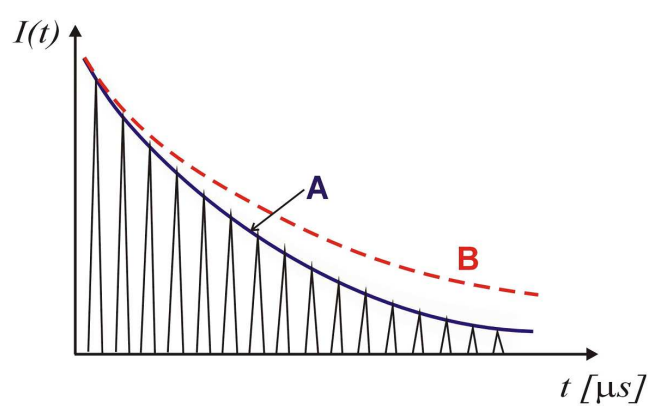

Fig. 3. The detected radiation at the output mirror.

$$
\frac{\mathrm{d} I}{\mathrm{~d} t}=I\left[\frac{c(1-R)}{L}+\alpha c\right],
$$

where $L$ is the cavity length, $R$ is the mirror reflectivity, $c$ is the light velocity and $\alpha$ is the absorption coefficient at the laser wavelength.

Solving Eq. (1), it is obtained

$$
I(t)=I_{0} \exp \left(-\left(\frac{(1-R)+\alpha L}{L}\right) t\right)=I_{0} \mathrm{e}^{-t / \tau},
$$

where $I_{0}$ is the magnitude of the introduced light, $\tau$ is the cavity decay time.

The cavity decay time is given by

$$
\tau=\frac{L}{c[(1-R)+\alpha L]} .
$$

When the cavity is empty $(\alpha=0)$ the determining decay time is given by the reflectivity $(R)$ of the cavity mirrors and the cavity length $(L)$

$$
\tau_{0}=\frac{L}{c(1-R)} .
$$

By combining Eqs. (3) and (4) the absorption coefficient is determined by

$$
\alpha(\lambda)=\frac{L}{c}\left(\frac{1}{\tau(\lambda)}-\frac{1}{\tau_{0}(\lambda)}\right) .
$$

CRDS method is based on the determination of the decay time of the laser light leaking out from an optical cavity. Knowledge about the decay time of the empty cavity $\tau_{0}(\lambda)$ and the cavity filled with the absorbing gas $\tau(\lambda)$, respectively, allows to determine the concentration of absorbing gas.

It is possible to use the measured absorption strength to estimate the concentration $(N)$ of $\mathrm{NO}_{2}$ because

$$
\alpha=\sigma N,
$$

where $\sigma$ is the absorption cross-section.

Detection limit of the CRDS method depends on the laser beam quality, the optical alignment, the mechanical and thermal stabilities, aerosols and Rayleigh scattering and the mirrors contamination.

$>$ From (5) and (6) the absorber concentration can be calculated

$$
N=\frac{L}{\sigma c}\left(\frac{1}{\tau}-\frac{1}{\tau_{0}}\right)=\frac{L}{\sigma c \tau}\left(\frac{\tau_{0}-\tau}{\tau_{0}}\right),
$$

Assuming that the relative precision of the CRDS time determination is equal to $\gamma=\left(\tau_{0}-\tau\right) / \tau_{0}$, one achieves that detectable concentration limit $N_{\text {Limit }}$ is described by the formula

$$
N_{\text {Limit }}=\frac{\gamma L}{c \sigma \tau_{0}} .
$$

It depends on the absorption cross-section, the relative precision of the CRDS time determination and the empty cavity decay time.

\section{Introduction to CEAS method}

Cavity enhanced absorption spectroscopy (CEAS) is a modification of CRDS technique. It is based on off-axis arrangement of the optical cavity. CEAS technique was described by Engeln in 1998 [2, 3]. In this configuration, the light is repeatedly reflected by the mirrors (like in multipass cells - Fig. 4), however, the reflection points are spatially separated.

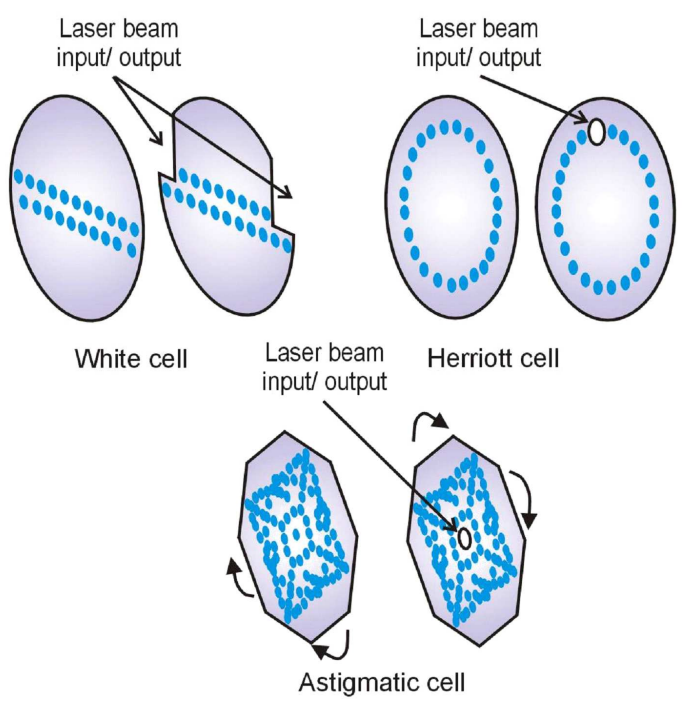

Fig. 4. Example of commonly used optical multipass cell.

CEAS has becoming a widely used technique in the optical absorption analysis of atoms and molecules. The technique allows the determination of total optical losses within a closed cavity comprised of two mirrors. CEAS is a technique capable of making quantitative diagnostic of trace species in a gaseous environment.

The off-axis design eliminates optical feedback from the cavity to the light source. Such arrangement causes that the light is many times reflected by the mirrors and it fills the whole volume of the cavity. In comparison with ordinary CDRS, the integrating spectroscopy with off-axis cavity adjusting is much less sensitive for the cavity misalignment caused, e.g., by the refractive index fluctuations due to turbulences or small mechanical instabilities. Moreover, in the CRDS method to ensure appropriate storing of light in the resonator, the frequency of probing laser mode must be well matched to a longitudinal cavity mode. Therefore, when single mode lasers are used, a 
good electronic stabilization and synchronization of the cavity and the laser frequency are necessary.

Furthermore, when the spectra of the absorbing species that fill the cavity consist of narrowband resonances, both the laser frequency and the cavity mode must be well matched to the absorption line.

Additionally, when a precise scanning over such sharp, narrowband spectrum is required, tuning and stabilization of the system provide difficult problems. These problems do not exist in CEAS technique, since due to multiply pass of light through the resonator before the beam overlapping the mode structure does not occur or is very weak.

\section{Experimental setup of CEAS sensor}

In CEAS method, the pulse of the laser light is injected into optical cavity (resonator) equipped with spherical and high reflective mirrors. The pulse yields to multiple reflections in the resonator. After each reflection, part of the laser light leaves the resonator because of lack of $100 \%$ mirrors reflectivities. The part of light outgoing from cavity is registered by a photodetector (photomultiplier tube - PMT). The electric signal from PMT can be observed and archived e.g. by the digital oscilloscope. The experimental setup of CEAS sensor is presented in Fig. 5.

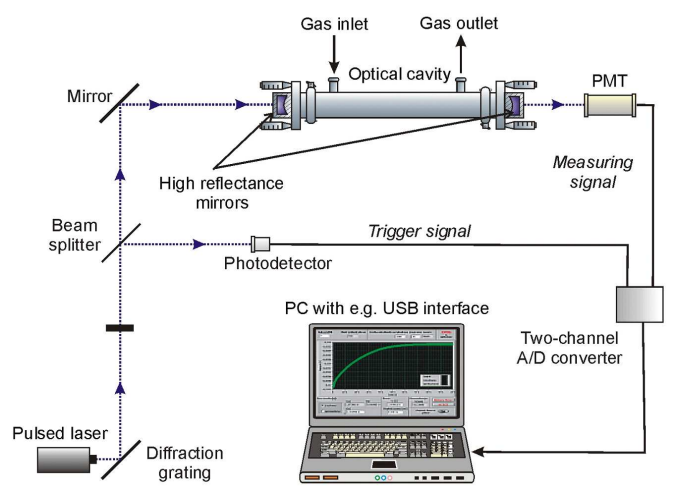

Fig. 5. Scheme of experimental setup.

The optical signal from the cavity is registered by a photodetector. It is often a photomultiplier. Nowadays, photomultipliers are characterized by high gain, high speed, low dark current and small dimensions. In the signal detection system, the most important parameter is signal to noise ratio $(\mathrm{S} / \mathrm{N})$. The ratio is determined by parameters of the first stage of preamp and features of digital signal processing (DSP) system (Fig. 6).

\section{Experimental results}

\section{1. $\mathrm{NO}_{2}$ detection}

Spectroscopy is widely used for monitoring of pollutant gases in the atmosphere. Nitrogen dioxide is one of the

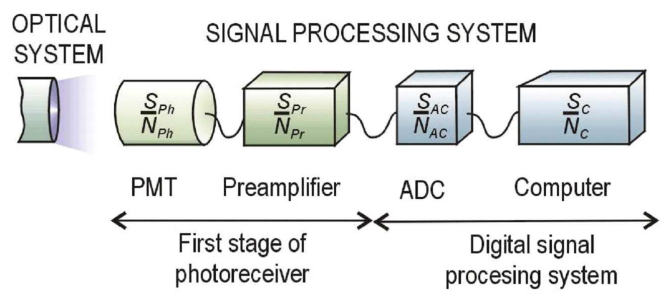

Fig. 6. Scheme of signal processing system used in CEAS optoelectronic sensor.

most important factor of atmospheric quality. Usually $\mathrm{NO}_{2}$ is detected by using the methods based on chemiluminescence, with single ppb sensitivity. Recent development of GaN and InGaN laser diodes provides opportunity to construct integrated optoelectronic gas sensor.

The optical resonator consisted of two concave mirrors whose reflectivity reached value of 0.99998 at the wavelength of interest (Fig. 7).

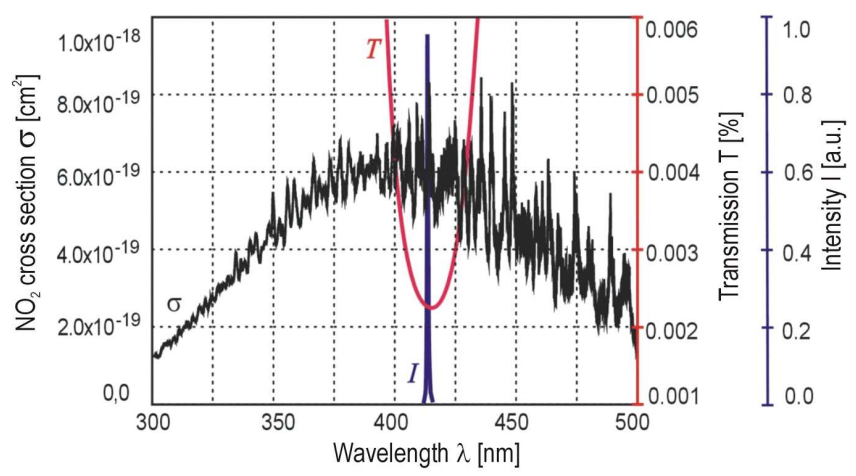

Fig. 7. Spectra of: laser radiation, mirrors reflectance, and $\mathrm{NO}_{2}$ absorption cross-section [4].

Since the length of the cavity was about $60 \mathrm{~cm}$, it provided to observe the decay time even to $25 \mu \mathrm{s}$. As a light source a $500 \mathrm{~mW}$ pulsed laser was used. It was working at the wavelength of $414 \mathrm{~nm}$ with repetition rate of $1 \mathrm{kHz}$ and pulse duration of $50 \mathrm{~ns}$. The laser radiation was directed to the cavity using the two mirrors. The cavity was equipped with gas inlet and outlet, which were connected with the gas mixing system. The system allowed for precision gas mixing and establishing ordered $\mathrm{NO}_{2}$ concentration [5].

The output signal was detected with a PMT equipped with the spectral filter eliminating interferences. The signal was observed and recorded with fast digital oscilloscope. The $\mathrm{NO}_{2}$ concentration was determined using Eq. (7). After averaging the signal of over 2048 pulses, about $\gamma=1 \%$ precision of the decay time determination was achieved, and after averaging of the signal of over 10000 laser pulses, precision about $\gamma=0.2 \%$ was achieved (Fig. 8).

According to Eq. (8) the detection limit better than $0.5 \mathrm{ppb}$ was obtained for $\sigma=6 \times 10^{-19} \mathrm{~cm}^{-2}[6-8]$. It 


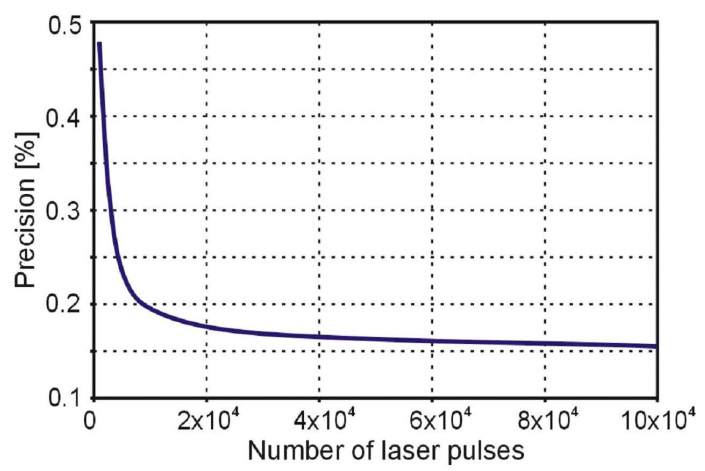

Fig. 8. Precision of decay time determination as a function of pulses number.

was noticed that, to achieve high value of $\tau_{0}$ and high precision of the decay time determination, the proper adjustment of the cavity is necessary. Moreover, the measurement with good detection limit also requires appropriate filtration of the investigated air. This is necessary in order to avoid the light scattering in the aerosol particles as well as the dust deposition on the mirror surfaces. When the concentration of aerosol particles in gas is similar to that in free atmosphere, the extinction of about $10^{-6} \mathrm{~m}^{-1}$ occurs, consequently at high sensitivity measurements, the losses due to the light scattering can be the same or larger than the absorption losses.

\section{2. $\mathrm{NO}_{3}$ detection}

The described sensor was also used to demonstrate nitrate $\left(\mathrm{NO}_{3}\right)$ absorption spectrum measurements. In this case a dye laser working in the $629 \mathrm{~nm}$ to $676 \mathrm{~nm}$ range was applied. The achieved results were comparable with $\mathrm{NO}_{3}$ reference data (Fig. 9) [8-10].

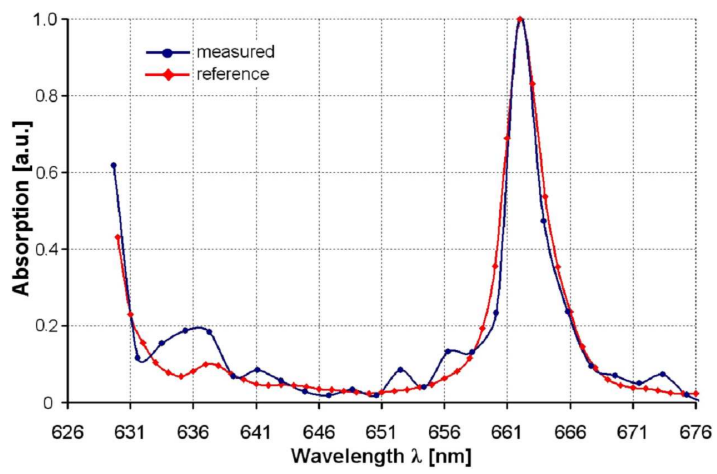

Fig. 9. Measured and reference $\mathrm{NO}_{3}$ absorption spectrum.

\section{Future work}

In the frame of the further work the sensor will be applied to nitric oxide (NO) detection. The NO absorption cross-section is presented in Fig. 10 [11].

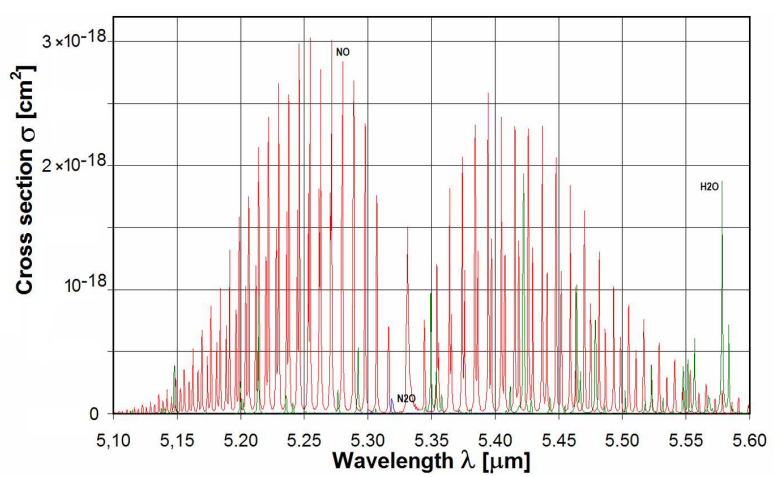

Fig. 10. Dependence NO absorption cross-section $\sigma$ on wavelength $\lambda$.

In the mentioned wavelength range, QCL laser (e.g. Alpes laser model \#sb1770DN) and photoreceiver with detector optimized for wavelength of interest ( $c a$. $5.25 \mu \mathrm{m})$ will be used. Such type of detectors are available from Vigo Systems (e.g. PVI-2TE-5 type). These detectors are produced using mercury cadmium telluride (MCT) epitaxial techniques and are equipped with thermoelectric coolers.

\section{Conclusions}

In the paper, $\mathrm{NO}_{x}$ optoelectronic sensor is described. The sensor operates basing on CEAS technique. It is one of the most sensitive techniques. This system ensures registration of low level signals, and the decay time measurements with the uncertainty below $0.2 \%$. The system consists of PMT (R7518, Hamamatsu), low noise transimpedance preamp (with AD8038, Analog Devices), and 12-bit digital signal processing circuit with the implemented coherent averaging technique. The features of a designed sensor show that it is possible to build a $\mathrm{NO}_{x}$ sensor with the sensitivity of sub-ppb level. The results of preliminary researches show that the system might be also applied to detection of other gas.

\section{Acknowledgments}

Authors thanks Prof. T. Stacewicz (Warsaw University) and Dr. A. Czyżewski (Institute of Applied Optics) for cooperation. The researches were funded by Polish Ministry of Science - grant no. R00-O0025/3.

\section{References}

[1] A. O'Keefe, D.A. Deacon, Rev. Sci. Instrum. 59, 2544 (1988).

[2] R. Engeln, G. Berden, R. Peeters, G. Meijer, Rev. Sci. Instrum. 69, 3763 (1998).

[3] V.L. Kasyutich, C.E. Canosa-Mas, C. Pfrang, S. Vaughan, R.P. Wayne, Appl. Phys. B 75, 755 (2002). 
[4] M.F. Merienne, A. Jenouvrier, B. Coquart, J. Atmos. Chem. 20, 281 (1995).

[5] J. Wojtas, A. Czyżewski, T. Stacewicz, Z. Bielecki, Opt. Appl. 36, 461 (2006).

[6] J. Wojtas, Z. Bielecki, Opto-Electron. Rev. 16, 44 (2008).

[7] Z. Bielecki, W. Kołosowski, G. Różański, J. Wojtas, Computational Methods and Experimental Measurements, WIT Press, Southampton 2007, p. 809.
[8] W.J. Marinelli, D.M. Svanson, H.S. Johnson, J. Chem. Phys. 76, 2864 (1982).

[9] A. Szpakowski, C. Tyszkiewicz, T. Pustelny, Acta Phys. Pol. A 114, A-237 (2008).

[10] T. Pustelny, I. Zielonka, C. Tyszkiewicz, P. Karasinski, B. Pustelna, Opto-Electron. Rev. 46, 221 (2006).

[11] http://www.epa.gov/ttn/emc/ftir/aedcdat1.html . 